\title{
ON ARITHMETICAL BOUNDS OF CHOW-FORMS
}

\author{
By
}

Tatsuji TANAKA

\begin{abstract}
In this paper, we will show that classical resultants-which are generalizations of Sylvester determinants-are closely related to Chow-forms of projective varieties. As a result, we will see that the maximal degree of the coefficients of a Chow-form in the coefficients of defining equations may be assumed to be bounded from above.
\end{abstract}

\section{Introduction}

If $V$ is an abelian variety embedded in $\boldsymbol{P}^{n}$ by using Theta functions, $V$ is defined by quadratic equations as coefficients admitting Theta constants ([I], [Mu1]). In this case, it seems to the author that Chow-forms of projective abelian varieties are explicitly described by using those Theta constants (cf. [Si]). Since Chow-forms play important roles in the arithmetical theory of the moduli theory of polarized abelian varieties and also they give homogeneous coordinates of closed points of the corresponding Chow variety, it seems to be worth to the author that we study explicit descriptions of Chow-forms. This paper may be considered to be an attempt to find explicit descriptions of Chow-forms.

If $V$ is an $r$-dimensional linear subvariety in $\boldsymbol{P}^{n}$, then $V$ has as its Chow-form the one whose coefficients are homogeneous polynomials of total degree $n-r$ in the coefficients of a system of defining linear forms. Our aim is a generalization of this fact. More explicitly, our main theorem is stated as follows: Let $V$ be any projective variety in $\boldsymbol{P}^{n}$. If the prime ideal of $V$ is generated by homogeneous polynomials $f_{1}, \ldots, f_{m}$ of degree bounded by $l$, then $V$ admits its Chow-form whose coefficients are given by homogeneous polynomials in the coefficients of $f_{1}, \ldots, f_{m}$ of total degree at most codim $V \times l^{\operatorname{codim} V-1}$ (Theorem 4.1)*.

\footnotetext{
* The main theorem was rewritten by suggestions from the referee. In the original manuscript it was stated as follows: "If the prime ideal of $V$ is generated by homogeneous polynomials $f_{1}, \ldots, f_{m}$ of like degree $l, \ldots$.

Received February 6, 2003.

Revised September 8, 2003.
} 
In order to prove the above theorem, we use the classical elimination theory, especially the theory of resultants. In fact, the elimination theory is one of methods by which we compute Chow-forms ([Vd2]). But actual computations of Chow-forms are far-reaching to us except some special varieties (e.g. [T]). As an example, let $V$ be an elliptic normal curve of degree 5 in $\boldsymbol{P}^{4} . V$ is defined by five quadratic forms with Theta constants as coefficients $([\mathrm{K}])$. We must compute the resultant ideal of the five quadratic forms and two generic linear forms. This computation is reduced to the computation of the resultant ideal of five quadratic forms in three variables. So we consider resultants of three quadratic forms in three variables. Such a resultant is known (e.g. [St]). The Chow-form of $V$ appears in those resultants as a factor. But the author can not explicitly determine the Chow-form of $V$.

An above mentioned resultant is a certain expression which is multihomogeneous in the coefficients of the given $n$ forms in $n$ variables and of certain multi-degree. We shall state its definition in 2.1. If $n=2$, that resultant is the same as an expansion of the Sylvester determinant.

About one hundred years ago, F. Mertens had proved fundamental results on the theory of resultants of $n$ forms in $n$ variables $([\mathrm{Me}])$. Our arguments are based on his results.

In $\S 2$, we prepare some results on the theory of resultants which are used in this paper. They had been proved in [Me].

In $\S 3$, we consider the case of complete intersection which is not necessarily irreducible and we show that the resultant is equal to a Chow-form (not necessarily irreducible). A key step for proving the main result is Theorem 3.1, which is essentially proved in [Me], except for the fact that the involving incidence correspondence preserves multiplicities. Especially, we see that the Chow-form of a variety which is a complete intersection is equal to a certain resultant.

In $\S 4$, we consider the general case which is not necessarily complete intersection and we shall prove the above mentioned main theorem. Here, our method is a reduction of the general case to the complete intersection case which will be treated in $\S 3$. That reduction is achieved by, to a given variety $V$, attaching a complete intersection cycle having $V$ as a component.

We shall work in a fixed universal domain of characteristic 0 and the prime field is denoted by $F$.

We shall mean by a $k$-variety ( $k$ a field) a variety embedded in $\boldsymbol{P}^{n}$ which is irreducible over $k$, but not necessarily absolutely irreducible. We shall call an absolutely irreducible variety simply a variety. 
The following notations will be used throughout this paper.

For sequences $u=\left(u_{0}, \ldots, u_{n}\right), v=\left(v_{0}, \ldots, v_{n}\right), \ldots$ of $n+1$ elements, we use classical notations

$$
\begin{aligned}
& u_{x}=u_{0} x_{0}+\cdots+u_{n} x_{n} \\
& v_{x}=v_{0} x_{0}+\cdots+v_{n} x_{n}
\end{aligned}
$$

for the corresponding linear forms, where $x_{0}, \ldots, x_{n}$ are independent variables.

By general (homogeneous) forms over a field $k$, we shall always mean homogeneous forms whose coefficients of all the monomials in $x_{0}, \ldots, x_{n}$ are independent variables over $k$.

For $n+1$ homogeneous forms $f_{0}, \ldots, f_{n}$ in the polynomial ring $k[x]=$ $k\left[x_{0}, \ldots, x_{n}\right]$, we denote the resultant (for the definition, cf. 2.1) by

$$
\mathscr{R}=\left[\begin{array}{lll}
f_{0} & \cdots & f_{n} \\
x_{0} & \cdots & x_{n}
\end{array}\right] .
$$

When $f_{1}, \ldots, f_{m}$ are homogeneous polynomials in $k[x]$, we shall always mean by the field of coefficients of $f_{1}, \ldots, f_{m}$ the subfield of $k$ generated by all the coefficients of them over the prime field $F$ and we shall denote it by $k_{0}$ (by writing the subscript 0 to $k$ ). If $f$ is a homogeneous form in $x_{0}, \ldots, x_{n}$ and $Q$ is a homogeneous polynomial in the coefficients of $f$, we denote by $\operatorname{deg}_{f} Q$ the degree of $Q$ in the coefficients of $f$.

$P_{V}$ is the Chow-form of a variety $V$.

\section{Preliminaries}

2.1. We recall the definition of resultants and some of their properties.

Let $\overline{f_{0}}, \ldots, \overline{f_{n}}$ be general forms in $x_{0}, \ldots, x_{n}$ over a field $k$. Let $m_{0}, \ldots, m_{n}$ be their respective degrees.

Let $A$ be the polynomial ring generated by all the coefficients of $\overline{f_{0}}, \ldots, \overline{f_{n}}$ over the prime field $F$. For each $i$, let $W_{i i}$ be the coefficient of $x_{i}^{m_{i}}$ in $\overline{f_{i}}$. We define

$$
P(\bar{f}):=W_{00}^{p_{0}} W_{11}^{p_{1}} \cdots W_{n n}^{p_{n}}
$$

where

$$
p=m_{0} m_{1} \cdots m_{n}, \quad p_{i}=\frac{p}{m_{i}}
$$

$P(\bar{f})$ is called the principal monomial of the sequence $\left\{\overline{f_{0}}, \ldots, \overline{f_{n}}\right\}$. 
Let $\mathscr{R}$ be a polynomial in $A$. Then we define $\mathscr{R}$ is the resultant of $\overline{f_{0}}, \ldots, \overline{f_{n}}$ w.r.t. $x_{0}, \ldots, x_{n}$

$$
\stackrel{\operatorname{def}}{\Longleftrightarrow}\left\{\begin{array}{l}
\left(\text { a) } \exists x_{i} \text { s.t. } \mathscr{R} x_{i}^{r} \in\left(\overline{f_{0}}, \ldots, \overline{f_{n}}\right) \text { the ideal in } A\left[x_{0}, \ldots, x_{n}\right] \text {, for some } r\right. \\
\text { (b) for each } i ; \mathscr{R} \text { is homogeneous in the coefficients of } \overline{f_{i}} \text { of } \\
\text { degree } p_{i}, \\
\text { (c) } \mathscr{R} \text { has the principal monomial } P(\bar{f}) \text { with coefficients } 1 .
\end{array}\right.
$$

There always exists one and only one resultant $([\mathrm{Hu}]$ or $[\mathrm{Me}], \mathrm{I})$. Henceforth, we denote this resultant $\mathscr{R}$ by

$$
\left[\begin{array}{lll}
\overline{f_{0}} & \cdots & \overline{f_{n}} \\
x_{0} & \cdots & x_{n}
\end{array}\right]
$$

If we change the orders of the sequences $\overline{f_{0}}, \ldots, \overline{f_{n}}$ and $x_{0}, \ldots, x_{n}$, then the corresponding resultant is the original resultant multiplied by the $p$-th powers of the signatures of the permutations $([\mathrm{Me}])$.

When $f_{0}, \ldots, f_{n}$ are homogeneous forms in the polynomial ring $k[x]$ whose degrees are $m_{0}, \ldots, m_{n}$, we call the unique specialization of (2) the resultant of $f_{0}, \ldots, f_{n}$ w.r.t. $x_{0}, \ldots, x_{n}$ and denote this resultant by

$$
\left[\begin{array}{lll}
f_{0} & \cdots & f_{n} \\
x_{0} & \cdots & x_{n}
\end{array}\right]
$$

In this case, we have

$$
V\left(f_{0}, \ldots, f_{n}\right) \neq \varnothing \Leftrightarrow\left[\begin{array}{lll}
f_{0} & \cdots & f_{n} \\
x_{0} & \cdots & x_{n}
\end{array}\right]=0
$$

where the algebraic set is the one lying in $\boldsymbol{P}^{n}(\S 23, \S 25$ in [Me], II).

Especially, when $f_{1}, \ldots, f_{n-r}$ are homogeneous forms in $k[x]$ and $u^{(0)}, \ldots, u^{(r)}$ are $r+1$ sequences of $n+1$ independent variables over $k$, we denote by $\mathscr{R}\left(u^{(0)}, \ldots, u^{(r)}\right)$ the resultant of the sequence $f_{1}, \ldots, f_{n-r}, u_{x}^{(1)}, \ldots, u_{x}^{(r)}, u_{x}^{(0)}$, i.e.,

$$
\mathscr{R}\left(u^{(0)}, \ldots, u^{(r)}\right)=\left[\begin{array}{ccc}
f_{1}, \ldots, f_{n-r}, & u_{x}^{(1)}, \ldots, u_{x}^{(r)}, & u_{x}^{(0)} \\
x_{0}, \ldots, x_{n-r-1}, & x_{n-r}, \ldots, x_{n-1}, & x_{n}
\end{array}\right] .
$$

$\mathscr{R}\left(\boldsymbol{u}^{(0)}, \ldots, \boldsymbol{u}^{(r)}\right)$ is expressed as a homogeneous form in the minors of order $r+1$ of the matrix

$$
\left(\begin{array}{ccc}
u_{0}^{(0)} & \cdots & u_{n}^{(0)} \\
\vdots & \cdots & \vdots \\
u_{0}^{(r)} & \cdots & u_{n}^{(r)}
\end{array}\right)
$$


of degree $m_{1} \cdots m_{n-r}(\S 10$ in [Me], I $)$. If we denote by $k_{0}$ the field of coefficients of $f_{1}, \ldots, f_{n-r}$, then we have $\mathscr{R}\left(u^{(0)}, \ldots, u^{(r)}\right) \in k_{0}\left[u^{(0)}, \ldots, u^{(r)}\right]$.

2.2. When $\overline{f_{n}}=u_{x}=u_{0} x_{0}+\cdots+u_{n} x_{n}$ is a general linear form, instead of $\mathscr{R}$ and $p$ of (1), we use the notations:

$$
\begin{aligned}
\Theta=\Theta(u) & :=\left[\begin{array}{llll}
\overline{f_{0}} & \cdots & \overline{f_{n-1}} & u_{x} \\
x_{0} & \cdots & x_{n-1} & x_{n}
\end{array}\right], \\
v & :=m_{0} m_{1} \cdots m_{n-1}
\end{aligned}
$$

Then

$$
\operatorname{deg}_{\overline{f_{i}}} \Theta=\frac{v}{m_{i}} \quad(0 \leq i \leq n-1), \quad \operatorname{deg}_{u} \Theta=v .
$$

The following theorem ( $\S 22$ in $[\mathrm{Me}]$, II) is used in the proof of Theorem 3.1.

THEOREM 2.1. Let $f_{0}, \ldots, f_{n-1}$ be homogeneous forms in $k[x]$ of degree $m_{0}, \ldots, m_{n-1}$. Assume that $\Theta=\left[\begin{array}{cccc}f_{0} & \cdots & f_{n-1} & u_{x} \\ x_{0} & \cdots & x_{n-1} & x_{n}\end{array}\right] \not \equiv 0$ in $u_{0}, \ldots, u_{n}$. Then, over the algebraic closure $\overline{k_{0}}$ of the field of coefficients $k_{0}$ of the $f_{i}, \Theta$ is decomposed into a product

$$
\Theta=c \prod_{i=1}^{v}\left(\sum_{j=0}^{n} a_{i j} u_{j}\right)
$$

of $v$ linear forms in $u_{0}, \ldots, u_{n}$, where $c$ is a non-zero element of $k_{0}$.

Moreover, we have

$$
V\left(f_{1}, \ldots, f_{n-1}\right)=\left\{\left(a_{i 0}, \ldots, a_{i n}\right) \mid 1 \leq i \leq v\right\} .
$$

We note that the $v$ points $\left(a_{i 0}, \ldots, a_{i n}\right)$ are not necessarily distinct.

2.3. In $\S 4$, we use the following theorem ( $\S 11$ in $[\mathrm{Me}]$, I ):

THEOREM 2.2. Let $k, n$ and $m$ be integers s.t. $0<k<n+1 \leq m$ and let $\phi_{1}, \ldots, \phi_{m}$ be $m$ general forms of $x_{0}, \ldots, x_{n}$ of degree $\mu$. We define $k$ homogeneous forms $g_{1}, \ldots, g_{k}$ by

$$
\left(\begin{array}{c}
g_{1} \\
\vdots \\
g_{k}
\end{array}\right):=\left(\begin{array}{ccc}
c_{11} & \cdots & c_{1 m} \\
& \cdots & \\
c_{k 1} & \cdots & c_{k m}
\end{array}\right)\left(\begin{array}{c}
\phi_{1} \\
\vdots \\
\phi_{m}
\end{array}\right)
$$


where the $c_{i j}$ are indeterminates. Moreover, we take $n-k+1$ generic linear forms $u_{x}^{(0)}, \ldots, u_{x}^{(n-k)}$. Then the resultant

$$
\left[\begin{array}{cc}
g_{1}, \ldots, g_{k}, & u_{x}^{(0)}, \ldots, u_{x}^{(n-k)} \\
x_{0}, \ldots, x_{k-1}, & x_{k}, \ldots, x_{n}
\end{array}\right]
$$

is a polynomial in the minors of order $k$ of the matrix

$$
\left(\begin{array}{ccc}
c_{11} & \cdots & c_{1 m} \\
\vdots & & \vdots \\
c_{k 1} & \cdots & c_{k m}
\end{array}\right) .
$$

\section{Resultants and Chow-forms}

In this section, we shall prove that if an $r$-dimensional variety $V$ is a complete intersection, then its Chow-form $P_{V}$ coincides with the resultant of the defining equations of $V$ and $r+1$ general linear forms.

3.1. As a background of our main theme in this paper, we recall the following: the field generated by the Chow-point of a projective variety over the prime field $F$ is the smallest field of definition for that variety, and hence if $V$ is a variety of $\boldsymbol{P}^{n}$ over $k$ with the prime ideal $\mathfrak{P}$, and $\boldsymbol{P}_{V}$ is the Chow-form of $V$, then we can assume that the coefficients of $P_{V}$ are given by polynomials of the coefficients of homogeneous forms which constitute a basis of $\mathfrak{B}$.

3.2. Before we consider the case of complete intersections, we review the definition of the Chow-form $P_{\mathfrak{U}}$ of an unmixed homogeneous ideal $\mathfrak{A}$.

Let $k$ be a field in the universal domain and $R=k\left[x_{0}, \ldots, x_{n}\right]$ be the polynomial ring over $k$.

For any $k$-variety $V$ which is not necessarily absolutely irreducible, its Chowform $P_{V}$ can be defined. $P_{V}$ is irreducible over $k$.

Let $\mathfrak{p}$ be the homogeneous prime ideal in $R$ of a $k$-variety $V$, and we put $\mathfrak{p}^{\prime}=\mathfrak{p} \otimes_{k} \bar{k}$. If $\mathfrak{p}_{1}, \ldots, \mathfrak{p}_{m}$ are the prime ideals of $\mathfrak{p}^{\prime}$ and $V_{i}$ is the variety (absolutely irreducible) defined by $\mathfrak{p}_{i}$ for each $i$, then, by definition (since we have assumed the characteristic to be 0$)$, the cycle $Z\left(\mathfrak{p}^{\prime}\right)$ of $\mathfrak{p}^{\prime}$ is

$$
Z\left(\mathfrak{p}^{\prime}\right)=V_{1}+\cdots+V_{m} .
$$

If we denote by $P_{i}$ the Chow-form of $V_{i}$, then we have

$$
P_{V}=P_{1} \cdots P_{m} .
$$


This factorization is computed in the algebraic closure $\bar{k}$ (over some finitely generated extension of $k$ ).

In this case, we define

$$
\operatorname{deg} V:=\operatorname{deg} V_{1}+\cdots+\operatorname{deg} V_{m} .
$$

If $\operatorname{dim} V=r$ and $\operatorname{deg} V=d$, then the Chow-form $P_{V}=P_{V}\left(u^{(0)}, \ldots, u^{(r)}\right)$ of $V$ is a multi-homogeneous form in the $u^{(i)}$ of degree $d$, where $u^{(0)}, \ldots, u^{(r)}$ are $r+1$ sequences of $n+1$ independent variables over $k$.

Let $\mathfrak{A}$ be a homogeneous ideal in $R$ which is unmixed. Then the cycle $Z(\mathfrak{A} ; k)$ of $\mathfrak{U}$ over $k$ is defined as follows: if $\mathfrak{p}_{1}, \ldots, \mathfrak{p}_{l}$ are the (homogeneous) prime ideals of $\mathfrak{A}$, then

$$
Z(\mathfrak{U} ; k):=\text { length }_{R_{\mathfrak{p}_{1}}}(R / \mathfrak{U})_{\mathfrak{p}_{1}} \cdot V_{1}+\cdots+\text { length }_{R_{\mathfrak{p}_{l}}}(R / \mathfrak{U})_{\mathfrak{p}_{l}} \cdot V_{l},
$$

where the $V_{i}$ are $k$-varieties defined by the $\mathfrak{p}_{i}$.

Now, we assume that $\mathfrak{U}$ is generated by $n-r$ forms $f_{1}, \ldots, f_{n-r}$ in $R$ and is of height $n-r$. Then $\mathfrak{U}$ is unmixed. Let $k_{0}$ be the field of coefficients of $f_{1}, \ldots, f_{n-r}$. Let

$$
Z\left(\mathfrak{U} ; k_{0}\right)=a_{1} V_{1}+\cdots+a_{l} V_{l}
$$

be the $r$-cycle of $\mathfrak{U}$ in $\boldsymbol{P}^{n}$ defined over $k_{0}$, where $V_{1}, \ldots, V_{l}$ are $k_{0}$-irreducible varieties. Then we define the Chow-form $P_{\mathfrak{A}}$ of the ideal $\mathfrak{A}$ by

$$
P_{\mathfrak{Q}}:=P_{1}^{a_{1}} \cdots P_{l}^{a_{l}}
$$

with $P_{i}$ the Chow-form of $V_{i}$. Since the Chow-form of an absolutely irreducible variety is absolutely irreducible, $P_{\mathfrak{A}}$ is uniquely determined by the given ideal $\mathfrak{A}$.

3.3. Our key step is the following theorem:

THEOREM 3.1. Let $f_{1}, \ldots, f_{n-r}$ be $n-r$ homogeneous forms in $k[x]$ for some field $k$. Suppose that the ideal $\mathfrak{U}=\left(f_{1}, \ldots, f_{n-r}\right)$ of $k[x]$ is of height $n-r$. Then we have

$$
\mathscr{R}\left(u^{(0)}, \ldots, u^{(r)}\right)=P_{\mathfrak{A}} .
$$

Moreover, if $k_{0}$ is the field of coefficients of $f_{1}, \ldots, f_{n-r}$ and

$$
Z\left(\mathfrak{U} ; k_{0}\right)=a_{1} V_{1}+\cdots+a_{l} V_{l}
$$

then, in $k_{0}\left[u^{(0)}, \ldots, u^{(r)}\right]$, we have a decomposition

$$
\mathscr{R}\left(u^{(0)}, \ldots, u^{(r)}\right)=P_{1}^{a_{1}} \cdots P_{l}^{a_{l}}, \quad P_{i}=P_{V_{i}} .
$$


Proof. It suffices to prove the second part of the claim. First we show that the resultant $\mathscr{R}\left(u^{(0)}, \ldots, u^{(r)}\right)$ is the Chow-form of an $r$-cycle in $\boldsymbol{P}^{n}$. For this, we go back to the classical definition of a Chow-form and we check the conditions $([\mathrm{H}-\mathrm{P}]$ or $[\mathrm{Sa}])$ for a given multi-homogeneous form to be a Chow-form.

The resultant $\mathscr{R}\left(u^{(0)}, \ldots, u^{(r)}\right)$ is multi-homogeneous in $u^{(0)}, \ldots, u^{(r)}$, whose degree in $u^{(i)}$ is $m_{1} \cdots m_{n-r}$ and $\mathscr{R}\left(u^{(0)}, \ldots, u^{(r)}\right) \in k_{0}\left[u^{(0)}, \ldots, u^{(r)}\right]$.

We consider $\mathscr{R}\left(u^{(0)}, \ldots, u^{(r)}\right)$ as a form in $u^{(0)}$ and we denote it by $F\left(u^{(0)}\right)$. $F\left(u^{(0)}\right)$ is equal to $\Theta\left(u^{(0)}\right)$ in 2.2 for the sequence $f_{1}, \ldots, f_{n-r}, u_{x}^{(1)}, \ldots, u_{x}^{(r)}$.

$$
L \stackrel{\text { put }}{=} V\left(u_{x}^{(0)}, u_{x}^{(1)}, \ldots, u_{x}^{(r)}\right)
$$

$L$ is a linear subspace generic over $k$ of dimension $n-(r+1)$.

From the assumption, $V(\mathfrak{U})$ is of pure dimension $r$. Hence $V(\mathfrak{U}) \cap L=\varnothing$. Thus we have

$$
V\left(f_{1}, \ldots, f_{n-r}, u_{x}^{(0)}, u_{x}^{(1)}, \ldots, u_{x}^{(r)}\right)=\varnothing .
$$

Hence, $\Theta\left(u^{(0)}\right) \not \equiv 0$. Therefore, by Theorem 2.1 in 2.2, $F\left(u^{(0)}\right)$ decomposes into linear forms in $u^{(0)}$ over $\overline{k_{0}\left(u^{(1)}, \ldots, u^{(r)}\right)}$.

Now

$$
F\left(u^{(0)}\right) \stackrel{\text { put }}{=} c \prod_{q=1}^{d}\left(\sum_{i=0}^{n} x_{i}^{(q)} u_{i}^{(0)}\right)
$$

where $c, x_{i}^{(q)} \in \overline{k_{0}\left(u^{(1)}, \ldots, u^{(r)}\right)}$ and $d=\operatorname{deg} f_{1} \cdots \operatorname{deg} f_{n-r}$.

For the points $x^{(q)}=\left(x_{0}^{(q)}, \ldots, x_{n}^{(q)}\right)$, by Theorem 2.1, we have

$$
\sum_{i=0}^{n} x_{i}^{(q)} u_{i}^{(s)}=0 \quad(1 \leq \forall s \leq r, 1 \leq \forall q \leq d) .
$$

Suppose that $r+1$ hyperplanes $H_{s}=V\left(\sum_{i=0}^{n} v_{i}^{(s)} x_{i}\right)(0 \leq s \leq r)$ pass through some point $x^{(q)}$.

Then $x^{(q)}$ is a common solution of $f_{1}=\cdots=f_{n-r}=0$ and hence

$$
f_{1}=\cdots=f_{n-r}=v_{x}^{(1)}=\cdots=v_{x}^{(r)}=v_{x}^{(0)}=0
$$

has a non-trivial common solution $x^{(q)}$. Thus we have

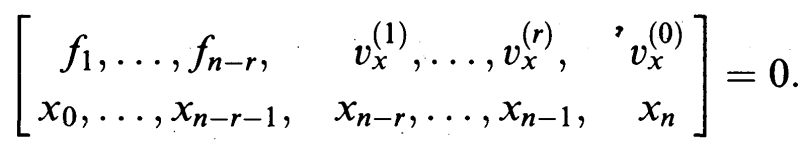

Since the left side is $\mathscr{R}\left(v^{(0)}, \ldots, v^{(r)}\right), \mathscr{R}\left(v^{(0)}, \ldots, v^{(r)}\right)=0$.

Therefore $\mathscr{R}\left(u^{(0)}, u^{(1)}, \ldots, u^{(r)}\right)$ is the Chow-form of a cycle in $\boldsymbol{P}^{n}([\mathrm{H}-\mathrm{P}])$. Next we show that this resultant is equal to the Chow-form $P_{\mathfrak{U}}$ of the ideal $\mathfrak{A}$. 
For this, let $\check{\boldsymbol{P}}$ be the dual projective space of $\boldsymbol{P}^{n}$. We consider the incidence correspondence

$$
S \stackrel{\text { put }}{=}\left\{\left(v^{(0)}, \ldots, v^{(r)}\right) \in(\check{\boldsymbol{P}})^{r+1} \mid V(\mathfrak{A}) \cap\left(\bigcap_{j=0}^{r} V\left(\sum_{i=0}^{n} v_{i}^{(j)} x_{i}\right)\right) \neq \varnothing\right\}
$$

and

$$
S_{i} \stackrel{\text { put }}{=}\left\{\left(v^{(0)}, \ldots, v^{(r)}\right) \in(\check{\boldsymbol{P}})^{r+1} \mid V_{i} \cap\left(\bigcap_{j=0}^{r} V\left(\sum_{i=0}^{n} v_{i}^{(j)} x_{i}\right)\right) \neq \varnothing\right\} \quad(1 \leq i \leq l) .
$$

Since $V(\mathfrak{A})=V_{1} \cup \cdots \cup V_{l}$, we have

$$
S=S_{1} \cup \cdots \cup S_{l} \text {. }
$$

Thus, $S$ is a closed set of pure codimension 1 .

Since $S$ is defined by $\mathscr{R}\left(u^{(0)}, \ldots, u^{(r)}\right)$, the irreducible components of $S$ are defined by the irreducible factors of $\mathscr{R}\left(u^{(0)}, \ldots, u^{(r)}\right)$.

While, each $S_{i}$ is defined by the Chow-form $P_{i}$ of the component $V_{i}$ of $\mathfrak{A}$. Therefore for some integers $a_{i}^{\prime}$, we have

$$
\mathscr{R}\left(u^{(0)}, \ldots, u^{(r)}\right)=P_{1}^{a_{1}^{\prime}} \cdots P_{l}^{a_{1}^{\prime}}
$$

Here, for each $i, a_{i}^{\prime}$ is equal to the multiplicity of the component $S_{i}$.

Finally we must show that $a_{i}^{\prime}=a_{i}$. We recall that Chow-forms are considered as effective Cartier divisors on a certain grassmannian. Let $\mathcal{O}_{Z}$ be the structure sheaf $\mathcal{O}_{Z}$ of the closed subscheme $Z$ defined by the homogeneous ideal $\mathfrak{A}$ in $\boldsymbol{P}^{n}$. Then $S_{i}$ is a component of $\operatorname{Supp}\left(T_{t}\left(\mathcal{O}_{Z}(m)\right)\right.$, where $t=n-r-1$ and $T_{t}\left(\mathcal{O}_{Z}(m)\right)$ is the $t$-space Transform of $\mathcal{O}_{Z}(m)([\mathrm{F}])$. Under this set-up, we have

$$
a_{i}^{\prime}=\text { the multiplicity of the induced reduced closed subscheme of } S_{i} \text {. }
$$

Since the incidence correspondence $S$ preserves the multiplicities (Lemma 10.3 in [F] and $\S 3$ of Chap. 5 in [Mu2]), the right side of the above equality is $a_{i}$. Hence we get $a_{i}^{\prime}=a_{i}$.

Q.E.D.

COROllaRY 3.2. Let $V$ be a projective variety of $\boldsymbol{P}^{n}$ of dimension $r$.

Assume that $V$ is a complete intersection defined by the prime ideal $\left(f_{1}, \ldots, f_{n-r}\right)$. Then the resultant

$$
\left[\begin{array}{ccccccc}
f_{1} & \cdots & f_{n-r} & u_{x}^{(1)} & \cdots & u_{x}^{(r)} & u_{x}^{(0)} \\
x_{0} & \cdots & x_{n-r-1} & x_{n-r} & \cdots & x_{n-1} & x_{n}
\end{array}\right]
$$

is the Chow-form $P_{V}$ of $V$. 
Hence, in this case, a system of homogeneous coordinates of the Chow point $c(V)$ of $V$ is given by multi-homogeneous forms in the coefficients of $f_{1}, \ldots, f_{n-r}$, whose degree in the coefficients of $f_{i}$ is $d / m_{i}$, where $d=\operatorname{deg} V, m_{i}=\operatorname{deg} f_{i}$. This is the case which we treated in [T].

\section{Main Theorem}

In this section, we consider a variety (absolutely irreducible) which is not necessarily a complete intersection.

4.1. We note that if $V$ is a variety of $\boldsymbol{P}^{n}$ over $k$ with the prime ideal $\mathfrak{P}$, and $P_{V}$ is the Chow-form of $V$, then the coefficients of $P_{V}$ are multi-homogeneous in the coefficients of homogeneous forms which constitute a basis of $\mathfrak{P}$. In fact, let $\mathfrak{R}=\left\{D_{1}, \ldots, D_{h}\right\}$ be the resultant system of a basis $f_{1}, \ldots, f_{m}$ of $\mathfrak{P}$ and generic linear forms $u_{x}^{(0)}, \ldots, u_{x}^{(r)}(r=\operatorname{dim} V)$. Then $\operatorname{gcd} \mathfrak{R}$ is a power of $P_{V}$ ([Vd2]). While, each $D_{i}$ is multi-homogeneous in the coefficients of $f_{1}, \ldots, f_{m}$ ([Vd1]) and by 3.1 , the coefficients of $P_{V}$ are polynomials of them. Thus our statement is proved.

4.2. As above, let $V$ be a projective variety over a field $k$ in $\boldsymbol{P}^{n}$ of dimension $r(<n-1)$ and of degree $d$ defined by the prime ideal $\mathfrak{P}=\left(f_{1}, \ldots, f_{m}\right)$. We suppose that the $f_{i}$ 's are of degree bounded by $l$.

Following Mertens $(\S 25,[\mathrm{Me}]$, II) we construct new homogeneous forms of degree $l$ as follows:

We may assume that

$$
\left\{f_{1}, \ldots, f_{h}\right\}=\left\{f_{i} \mid \operatorname{deg} f_{i}=l\right\}, \quad\left\{f_{h+1}, \ldots, f_{m}\right\}=\left\{f_{i} \mid \operatorname{deg} f_{i}<l\right\} .
$$

For each $i$, let $\mu_{i}$ be the degree of $f_{i}$. Then we define $f_{1}^{\prime}, \ldots, f_{s}^{\prime}$ by

$$
\left\{f_{1}^{\prime}, \ldots, f_{s}^{\prime}\right\}:=\text { the set consisting of }\left\{\begin{array}{l}
f_{1}, f_{2}, \ldots, f_{h} \\
x_{0}^{l-\mu_{h+1}} f_{h+1}, x_{1}^{l-\mu_{h+1}} f_{h+1}, \ldots, x_{n}^{l-\mu_{h+1}} f_{h+1}, \\
x_{0}^{l-\mu_{h+2}} f_{h+2}, x_{1}^{l-\mu_{h+2}} f_{h+2}, \ldots, x_{n}^{l-\mu_{h+2}} f_{h+2}, \\
\ldots \ldots \\
x_{0}^{l-\mu_{m}} f_{m}, x_{1}^{l-\mu_{m}} f_{m}, \ldots, x_{n}^{l-\mu_{m}} f_{m} .
\end{array}\right.
$$

Let $\mathfrak{A}$ be the ideal $\left(f_{1}^{\prime}, \ldots, f_{s}^{\prime}\right)$ in $k[x]$.

By putting $k=n-r$, we apply Theorem 2.2 in 2.3 to the $s$ forms $f_{1}^{\prime}, \ldots, f_{s}^{\prime}$, i.e., 


$$
\left(\begin{array}{c}
g_{1} \\
\vdots \\
g_{n-r}
\end{array}\right):=\left(\begin{array}{ccc}
c_{11} & \cdots & c_{1 s} \\
& \cdots & \\
c_{n-r 1} & \cdots & c_{n-r s}
\end{array}\right)\left(\begin{array}{c}
f_{1}^{\prime} \\
\vdots \\
f_{s}^{\prime}
\end{array}\right)
$$

where the $c_{i j}$ are indeterminates.

We consider the resultant

$$
\mathscr{R}(g):=\left[\begin{array}{cc}
g_{1}, \ldots, g_{n-r}, & u_{x}^{(0)}, \ldots, u_{x}^{(r)} \\
x_{0}, \ldots, x_{n-r-1}, & x_{n-r}, \ldots, x_{n}
\end{array}\right] .
$$

$\mathscr{R}(g)$ is a polynomial in the minors of order $n-r$ of the matrix

$$
\left(\begin{array}{ccc}
c_{11} & \cdots & c_{1 s} \\
& \cdots & \\
c_{n-r 1} & \cdots & c_{n-r s}
\end{array}\right)
$$

In this case, we have $p=l^{n-r}, p_{i}=l^{n-r-1}(1 \leq i \leq n-r)$ and $\mathscr{R}(g)$ is homogeneous in the coefficients of $g_{i}$ of degree $l^{n-r-1}$.

Since $g_{i}=\sum_{j=1}^{s} c_{i j} f_{j}^{\prime}, \mathscr{R}(g)$ is homogeneous in $\left(c_{i 1}, \ldots, c_{i s}\right)$ of degree $l^{n-r-1}$ for each $i$.

$$
\mathscr{R}(g) \stackrel{\text { put }}{=} \sum_{|\alpha|=l^{n-r-1}} D_{\alpha} c^{\alpha}
$$

where the $c^{\alpha}$ are monomials in the determinants

$$
\left|\begin{array}{ccc}
c_{1 j_{1}} & \cdots & c_{1 j_{n-r}} \\
\vdots & & \vdots \\
c_{n-r j_{1}} & \cdots & c_{n-r j_{n-r}}
\end{array}\right| \quad\left(1 \leq j_{1}<\cdots<j_{n-r} \leq s\right)
$$

of degree $l^{n-r-1}$.

For convenience, we assume that $c^{\alpha}$ 's are standard monomials, i.e., they form a subsystem of a base of $\Gamma\left(G, \mathcal{O}_{G}\left(l^{n-r-1}\right)\right)$ where $G$ is the corresponding Grassmann variety $([\mathrm{H}-\mathrm{P}])$. Each $D_{\alpha}$ does not contain any $c_{i j}$.

4.3. Now we consider the algebraic set defined by $g_{1}, \ldots, g_{n-r}$. Let $k^{\prime}=k(c)$ be the field generated by $\left\{c_{i j}\right\}$ over $k \cdot \mathfrak{P}=\left(f_{1}, \ldots, f_{m}\right)$ is the above prime ideal of $V$ in $k[x]$ and $\mathfrak{U}=\left(f_{1}^{\prime}, \ldots, f_{s}^{\prime}\right)$ is the ideal. We denote by $\mathfrak{P}^{e}$ (resp. $\mathfrak{U}^{e}$ ) and $\mathfrak{U}^{\prime}$ the ideals generated by $f_{1}, \ldots, f_{m}$ (resp. $f_{1}^{\prime}, \ldots, f_{s}^{\prime}$ ) and $g_{1}, \ldots, g_{n-r}$ in $k^{\prime}[x]$. The ideal $\mathfrak{P}^{e}$ contains $\mathfrak{U}^{e}$ and hence also contains $\mathfrak{U}^{\prime}$. 
Lemma. The closed set $V\left(\mathfrak{U}^{\prime}\right)$ is of pure dimension $r$.

Proof. Since the algebraic set $V(\mathfrak{A})$ coincides with $V$, the ideal $\mathfrak{A}$ is of height $n-r$. Therefore we choose $n-r$ forms from the set $\left\{f_{1}^{\prime}, \ldots, f_{s}^{\prime}\right\}$ such that they form a $k[x]$-regular sequence. Say, $f_{h_{1}}^{\prime}, \ldots, f_{h_{n-r}}^{\prime}$ is such a $k[x]$-regular sequence. Let $R$ be the valuation ring in $\overline{k^{\prime}}$ of a place of $\overline{k^{\prime}}$ which is an extension of the specialization

$$
c_{i j} \rightarrow \delta_{j h_{i}} \quad(1 \leq i \leq n-r, 1 \leq j \leq s)
$$

over $k$. Then the specialization of $V\left(\mathfrak{U}^{\prime}\right)$ with respect to $R$ is $V\left(f_{h_{1}}^{\prime}, \ldots, f_{h_{n-r}}^{\prime}\right)$. Since the algebraic set $V\left(f_{h_{1}}^{\prime}, \ldots, f_{h_{n-r}}^{\prime}\right)$ has pure dimension $r, V\left(\mathfrak{U}^{\prime}\right)$ is also of pure dimension $r$.

Q.E.D.

We denote by $Z(V)$ the cycle $Z\left(\mathfrak{U}^{\prime}\right)$ determined by the ideal $\mathfrak{A}^{\prime}$.

4.4. We prove our main theorem:

THEOREM 4.1. Let $V$ be a projective variety of $\boldsymbol{P}^{n}$ of dimension $r<n-1$. Assume that $V$ is defined by $m$ homogeneous polynomials $f_{1}, \ldots, f_{m}$ of degree bounded by $l$. Then there exists a Chow-form $P_{V}$ of $V$ whose coefficients are homogeneous polynomials in the coefficients of $f_{1}, \ldots, f_{m}$ of total degree $(n-r) l^{n-r-1}$.

Proof. We use the above notations. By the lemma, $Z(V)$ is an $r$-cycle. Since $V$ is absolutely irreducible and the ideal $\mathfrak{A}^{\prime}$ is contained in $\mathfrak{P}^{e}, V=V\left(\mathfrak{P}^{e}\right)$ is a component of $Z(V)$. Hence $Z(V)$ is of the form $Z(V)=a V+E$ for some integer $a \geq 1$, where $E$ is the part in which $V$ is not a component.

Since the ideal $\mathfrak{U}^{\prime}$ is generated by $n-r$ forms and of height $n-r$, by Theorem 3.1, $\mathscr{R}(g)$ is equal to the Chow-form $P_{\mathfrak{U}^{\prime}}$ of the ideal $\mathfrak{U}^{\prime}$. Therefore, we get

$$
\mathscr{R}(g)=P_{V}^{a} \cdot P_{E}
$$

Let $k_{0}$ be the field generated by the coefficients of $f_{1}, \ldots, f_{m}$ over the prime field. We consider the cycle $Z\left(\mathfrak{U}^{\prime} ; k_{0}(c)\right)$ and we put

$$
Z\left(\mathfrak{U}^{\prime} ; k_{0}(c)\right)=a V+E .
$$

Then $\mathscr{R}(g) \in k_{0}[c]\left[u^{(0)}, \ldots, u^{(r)}\right]$ and $P_{V} \in k_{0}\left[u^{(0)}, \ldots, u^{(r)}\right]$. By Theorem 3.1, in $k_{0}[c]\left[u^{(0)}, \ldots, u^{(r)}\right]$, we have the factorization (9). 
By the condition (b) in the definition of the resultant (2.1), for each $i, \mathscr{R}(g)$ is homogeneous in the coefficients of $g_{i}$ of degree $l^{n-r-1}$.

Since each $g_{i}$ is linear in the coefficients of the $f_{j}$ 's, the total degree of $\mathscr{R}(g)$ in the coefficients of $f_{1}, \ldots, f_{m}$ is $(n-r) \times l^{n-r-1}$.

Therefore, by 4.1 , a Chow-form $P_{V}$ appearing in $\mathscr{R}(g)$ as a factor has the required property.

Q.E.D.

By (8), $P_{V}$ is a common factor of the $D_{\alpha}$ 's.

If the forms $f_{i}$ 's are of degree $l$, in (6) we can use the $f_{i}$ 's instead of the $f_{j}^{\prime \prime}$ 's. In this case, using the jacobian criterion $([\mathrm{Sa}])$, we see that $V$ is a simple component of the cycle $Z(V)$. Moreover, the equality (9) in the above proof also holds even if $V$ is a complete intersection, i.e., $m=n-r$. In fact, by $\S 13$, in [Me], I,

$$
\begin{aligned}
\mathscr{R}(g) & =\left[\begin{array}{cc}
g_{1}, \ldots, g_{n-r}, & u_{x}^{(0)}, \ldots, u_{x}^{(r)} \\
x_{0}, \ldots, x_{n-r-1}, & x_{n-r}, \ldots, x_{n}
\end{array}\right] \\
& =\left|\left(c_{i j}\right)\right|^{l^{n}}\left[\begin{array}{cc}
f_{1}, \ldots, f_{n-r}, & u_{x}^{(0)}, \ldots, u_{x}^{(r)} \\
x_{0}, \ldots, x_{n-r-1}, & x_{n-r}, \ldots, x_{n}
\end{array}\right]=\left|\left(c_{i j}\right)\right|^{l^{n}} P_{V} .
\end{aligned}
$$

In this case, since $E=0$, we consider $P_{E}$ as 1 . Thus the formula is valid up to a constant factor.

Finally, we give an example of the formula (9):

Let $V$ be the twisted cubic in $\boldsymbol{P}^{3}$ defined by the prime ideal

$$
\mathfrak{P}=\left(x z-y^{2}, y w-z^{2}, x w-y z\right) .
$$

We use the following notations:

$$
\begin{gathered}
p_{i j}=u_{i}^{(0)} u_{j}^{(1)}-u_{j}^{(0)} u_{i}^{(1)} \quad(0 \leq i<j \leq 3) \\
f_{1}:=x_{0} x_{2}-x_{1}^{2} \cdot \quad f_{2}:=x_{1} x_{3}-x_{2}^{2} \\
f_{3}:=x_{0} x_{3}-x_{1} x_{2} . \\
\left\{\begin{array}{l}
g_{1}:=c_{11} f_{1}+c_{12} f_{2}+c_{13} f_{3} \\
g_{2}:=c_{21} f_{1}+c_{22} f_{2}+c_{23} f_{3}
\end{array} .\right. \\
C_{i j} \stackrel{\text { put }}{=}\left|\begin{array}{ll}
c_{1 i} & c_{1 j} \\
c_{2 i} & c_{2 j}
\end{array}\right|
\end{gathered}
$$

Now, we take a set of indeterminates $U_{j}^{(i)}$ and denote by capitals $P_{i j}$ the corresponding expressions which result if in the Plücker coordinates $p_{i j}$ the $u_{j}^{(i)}$ 
are replaced by $U_{j}^{(i)}$. Then the Chow-form $P_{V}$ of $V$ and the above $\mathscr{R}(g)$ are given by

$$
\begin{gathered}
P_{V}=P_{03}^{3}+P_{02}^{2} P_{23}-P_{02} P_{03} P_{13}+P_{01} P_{13}^{2}-2 P_{01} P_{03} P_{23}-P_{01} P_{12} P_{23}, \\
\mathscr{R}(g)=P_{V} \cdot P_{01}^{4}\left(C_{12}^{2} P_{03}-C_{12} C_{13} P_{13}+C_{12} C_{23} P_{02}+C_{13}^{2} P_{23}-2 C_{13} C_{23} P_{12}+C_{23}^{2} P_{01}\right) .
\end{gathered}
$$

\section{Acknowledgement}

The author is highly grateful to the referee for valuable comments and suggestions, in accordance with which the author could improve the main theorem.

\section{References}

[F] Fogarty, J.: Truncated Hilbert functors, J. Reine und Angew. Math., 234, 65-88, 1969.

[H-P] Hodge, W. V. D.-Pedoe, D.: "Methods of Algebraic Geometry", Vol. II, Cambridge, 1968.

[Hu] Hurwitz, A.: Über die Trägheitsformen eines algebraischen Moduls, Annali die Mathematica. pura ed applics, serie III, t. 20, 113-151, 1913.

[I] Igusa, J.: "Theta functions", Grundlehren der math., Bd. 194, Springer-Verlag, 1972.

[K] Krazer, A.: "Lehrbuch der Thetafunktionen", Chelsea, New York, 1970.

[Me] Mertens, F.: Zur Theorie der Elimination. I-II. Sitzungsberichte der Kaiserlichen Akademie in Wien. Mathematisch-Naturwissenschaftliche Classe. Abt. II. a. Vol. 108, 1173-1228, Abt. II. b. Vol. 108, 1344-1386, 1899.

[Mul] Mumford, D.: Varieties defined by quadratic equations, Questioni sulle varieta algebraiche, Corsi dal C.L.M.E., Edizioni Cremonese, Roma, 1969.

[Mu2] Mumford, D.: “Geometric Invariant Theory”, Ergeb. Math., Bd. 34, Springer-Verlag, 1994.

[Sa] Samuel, P.: "Methodes d'Algebre Abstraite en Geometrie Algebrique”, Ergeb. Math., Bd. 4, Springer-Verlag, 1967.

[Si] Siegel, C. L.: Moduln Abelscher Funktionen, Gesammelte Abh. III, Springer-Verlag, 1964.

[St] Sturmfels, B.: Sparse elimination theory, in "Computational Algebraic Geometry and Commutative Algebra", Symposia Mathematica, 34, Cambridge, 1993.

[T] Tanaka, T.: On the Chow-forms of elliptic normal curves of degree 4, Tsukuba Journal of Mathematics, 24, 109-125, 2000.

[Vd1] van der Waerden, B. L.: "Moderne Algebra", II, Zweite Verbesserte Auflage, Springer-Verlag, 1940.

[Vd2] van der Waerden, B. L.: "Einführung in die algebraische Geometrie", Zweite Auflage, Grundlehren der math., Bd. 51, Springer-Verlag, 1973.

Tatsuji TANAKA

Department of Mathematics

Faculty of Sci. \& Engrg., Saga University

Saga 840-8502, Japan

e-mail: tanaka@ms.saga-u.ac.jp 